\title{
Development of an Active Vibration-Canceling System Using Inertial Force Generators"
}

\author{
Masaharu TAGAMI $^{* *}$, Tomoyuki YAMASHITA ${ }^{* *}$, Yasutaka TAGAWA ${ }^{* *}$, \\ Masashi YASUDA $^{* * *}$, Hirokazu HORA ${ }^{* * *}$, \\ Kenichi ENDO $^{* * *}$ and Yasuyuki NOGUCHI ${ }^{* * *}$ \\ **Dept. of Mechanical System Engineering, Tokyo University of Agriculture and Technology, \\ 2-24-16, Naka-Cho, Koganei, Tokyo 184-8588, Japan \\ E-mail: tagawa@cc.tuat.ac.jp \\ ***Tokkyokiki Corporation, \\ 10-133, Minami-Hatsushima-Cho, Amagasaki, Hyogo 660-0833 Japan
}

\begin{abstract}
The density of components on precision devices such as semiconductor processors has been increasing recently. As a consequence, the acceptable vibration level of floors on which manufacturing equipment is installed is decreasing. Conversely, vibrations generated by the manufacturing equipment are increasing because manufactured objects are increasing in size. For these situations, active vibration isolation devices are usually used to isolate equipment from floor vibrations. However, the vibration control forces generated by active vibration isolation devices cause floor vibrations when these devices are used to isolate the large vibrations generated by manufacturing equipment. To solve this problem, a new vibration-canceling system using inertial-force generators is developed in this research. In addition, the effectiveness of the developed vibration-canceling system is confirmed via experiments.
\end{abstract}

Key words: Micro Vibration, Vibration Control, Active Vibration Isolation Device, Dual Model Matching, Acceleration Control

\section{Introduction}

Semiconductor exposure apparatus exposing circuit patterns to silicon wafers consists of a stage device and a projection optics system, and requires an environment isolated from vibrations on the floor on which it is installed because the circuit patterns are microscopic. Therefore, an active vibration isolation device is required to operate this apparatus ${ }^{(1),(2)}$.

The active vibration isolation device consists of a table supported by actuators, which are installed on the floor, and the active vibration isolation device achieves vibration isolation and vibration damping by controlling the actuators appropriately. The working speed of the stage device, which carries and positions the silicon wafer, has been increasing. In addition, the stage device has been growing in size along with the wafer size. As a result, the amplitude of the vibrations generated by the stage device has been increasing. Therefore, the active vibration isolation device has to generate a larger damping force. The reaction force of the large damping force vibrates the floor on which the device is installed, and this floor vibration negatively affects other production devices nearby ${ }^{(3)}$.

To solve this problem, we have developed an "Active Vibration-Canceling Device", which consists of a "canceller", a digital signal processor (DSP), and an accelerometer ${ }^{(4)}$. The "canceller" is an inertial-force generator developed by authors. The active vibration-canceling system is used with the active vibration isolation device, and this

Received 16 Jan., 2008 (No. T2-07-0161) Japanese Original : Trans. Jpn. Soc. Mech. Eng., Vol.73, No.735, C (2007),

pp.2948-2954 (Received 27 Feb., 2007) [DOI: 10.1299/jsdd.2.507] 
system can cancel the reaction force of the stage device by using the inertial force generated by the canceller. By canceling the reaction force of the stage device, floor vibrations caused by the active vibration isolation device can be reduced, because the damping force generated by the actuators of the active vibration isolation device can be reduced. In this paper, the design of the active vibration-canceling system and its effects are shown in our experimental results.

\section{Active Vibration-Canceling System}

\subsection{Mechanism of Vibration-Canceling}

Figures 1 and 2 show the schematic view and the photograph of the active vibration-canceling system. The active vibration-canceling system is installed between the two parts of the table. The stage device, which handles the wafers, is installed at the center of the upper table. The active vibration-canceling system consists of a canceller, a DSP, and an accelerometer to measure the stage acceleration. The experimental system (Fig. 2) is $0.9 \mathrm{~m}$ high, $1.2 \mathrm{~m}$ wide, $1.2 \mathrm{~m}$ deep, and weighs $3300 \mathrm{~kg}$. There are two types of cancellers. One is a "vertical canceller," which can generate an inertial force vertical to the installation plane, while the other is a "horizontal canceller," which can generate an inertial force parallel to the installation plane. Both types of cancellers generate an inertial force by moving movable mass using a linear motor. The accelerometer is attached to each canceller's movable mass to form the acceleration control system. Using this acceleration control system, the canceller can generate inertial force of arbitrary magnitudes. Four vertical cancellers are installed at each corner of the table, and one horizontal canceller is installed at the center of the lower table (Fig. 2). In addition, all experiments are conducted considering the three degrees of freedom (DOF) on the plane, as shown in Fig. 1. Therefore, the motion in the direction of depth is ignored.

The mechanism of the active vibration-canceling system is explained through Fig. 1. If

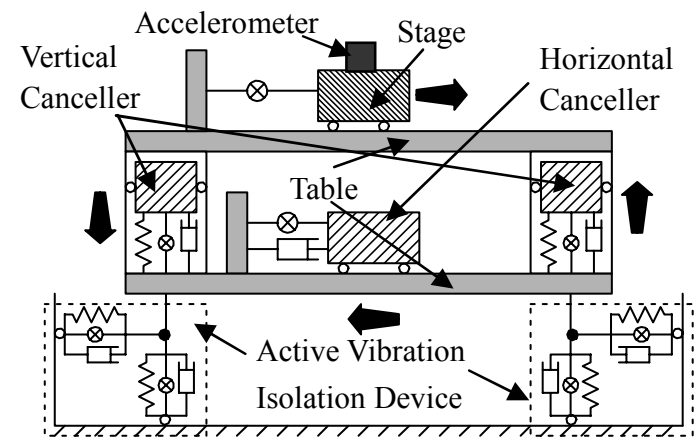

Fig. 1 Structure of the vibration-canceling system

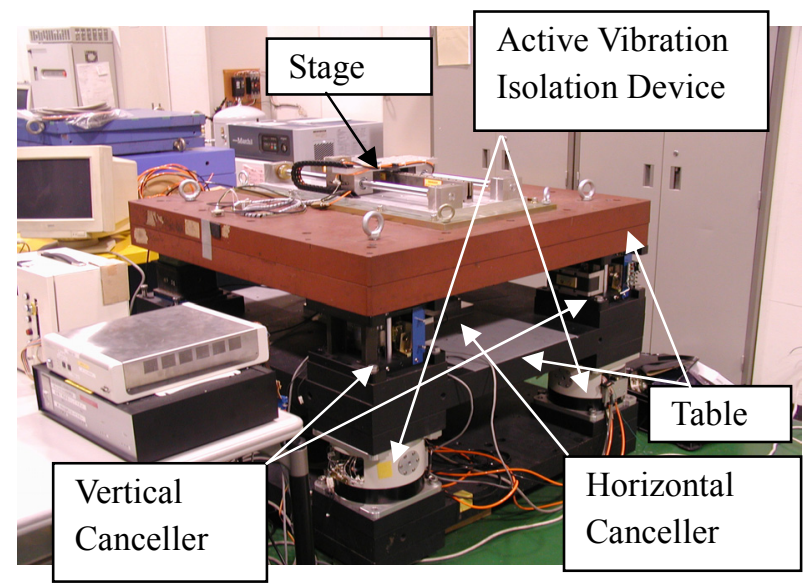

Fig. 2 Photograph of the vibration-canceling system 
the stage device installed on the table is accelerated, the inertial force acts on the table as a reaction force. This reaction force becomes a disturbance. Therefore, at first, the inertial force generated by the stage device can be estimated based on the acceleration of the stage device. Next, the cancellers generate inertial forces to cancel the disturbance (a reaction force and a reaction moment) via the estimated inertial force of the stage. In this way, the effect that the acceleration of the stage device has on the table can be cancelled. Therefore, the damping force generated by the active vibration isolation device can be reduced. As a result, the transmission of vibrations to the floor can be reduced. The difference between an active mass damper and this system is that the reaction force of the stage device is cancelled by the canceller, similar to a feedforward, using the estimated direct disturbance, which is estimated by an acceleration sensor on the stage device.

An example is as follows. When the stage device is accelerated to the right, the reaction force acts on the table in the left direction as a disturbance. To cancel this disturbance, the horizontal canceller's movable mass is accelerated to the left, which is the opposite direction of the stage device's acceleration. In this way, the horizontal canceller can provide the counter force to the force transferred by the stage device to the table. Then, if the force provided by the horizontal canceller is equal to the force transferred by the stage device to the table, the horizontal force acting on the table can be canceled. On the other hand, the anticlockwise moment caused by the stage device and the horizontal canceller acts on the table. However, a clockwise moment can be generated by accelerating the left side and the right side of the vertical canceller's movable masses in the downward and upward directions, respectively. Therefore, the disturbance moment can be canceled by appropriate control of the vertical canceller's inertial force. As mentioned above, in principle, the active vibration-canceling system can perfectly cancel vibrations generated by the stage device. We call this mechanism "vibration-canceling."

\subsection{Signal Flow of the Active Vibration-Canceling System}

Figure 3 shows the signal flow of the active vibration-canceling system. The stage device works independently from the active vibration-canceling system and can be controlled by a reference signal to its servo driver. The stage device's acceleration is measured by an accelerometer on the stage device and input to the DSP via the AD/C. The inertial force created by the stage device's motion can be estimated using this acceleration information. The reference signal for each canceller is determined by multiplying the acceleration of the stage device by a conversion matrix. The conversion matrix is a $5 \times 1$ real matrix. Based on the stage acceleration, this conversion matrix determines the reference acceleration that each canceller should generate to cancel the disturbance. This conversion

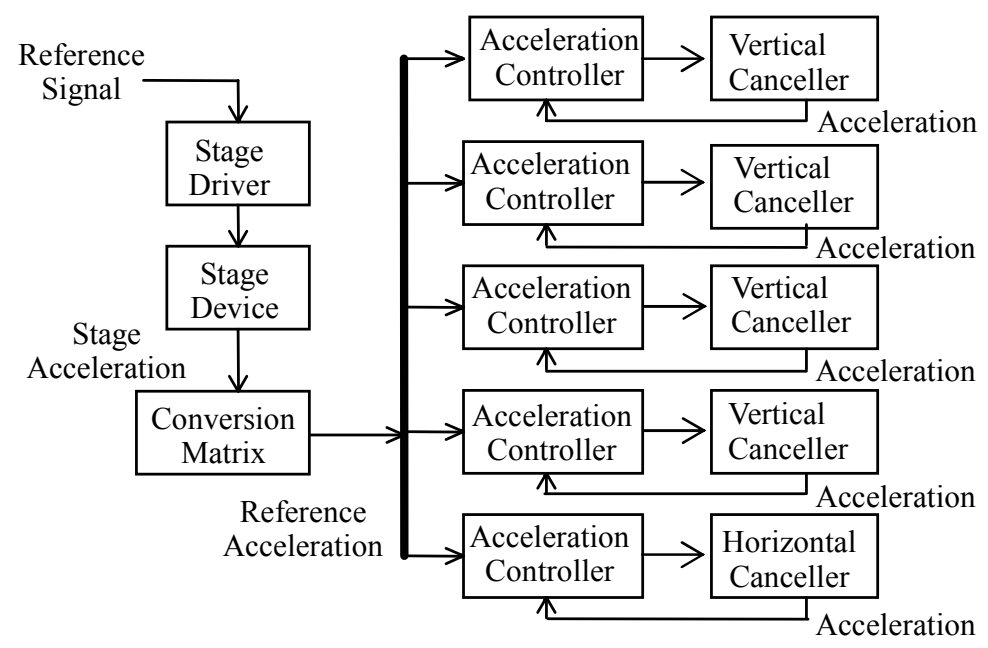

Fig. 3 Signal flow of the active vibration canceling system 
matrix is derived from the equilibrium of the force acting on the whole system including the active vibration isolation device. Each canceller's movable mass is controlled by the acceleration controller to generate the reference acceleration.

\subsection{Canceller}

As mentioned above, controlling the acceleration of the movable mass of each canceller is essential for vibration-canceling. In this subsection, therefore, the hardware and the acceleration controller of the canceller are described.

\subsubsection{Vertical Canceller}

Figure 4 shows a schematic view of the vertical canceller. The vertical canceller can generate an inertial force by driving the movable mass, which is supported by a spring and a linear guide, with a linear motor. The weight of the vertical canceller's movable mass is about $12 \mathrm{~kg}$. The vertical canceller's movable mass has an accelerometer, and an acceleration controller, which will be described later, can control the linear motor to generate desired acceleration in the wide frequency range. A plant analytical model of the vertical canceller is derived in order to design the acceleration controller. This model is given as the transfer function $P_{V}$, from the input signal for the linear motor driver, to the acceleration of the vertical canceller's movable mass [ Equation(1) ]. Since, the dynamics of the linear motor driver is much faster than other dynamics, it is ignored in Eq. (1).

$$
P_{V}=\frac{K_{v} s^{2}}{s^{2}+2 \zeta_{v} \omega_{n v} s+\omega_{n v}{ }^{2}}
$$

Here, $\omega_{n v}, \zeta_{v}$, and $K_{v}$ are the natural angular frequency, the damping ratio, and the gain of the vertical canceller, respectively. These parameters were identified via a frequency response experiment. In the frequency response experiment, a sine wave that sweeps from 0.5 to $200 \mathrm{~Hz}$ in $120 \mathrm{~s}$ was used as the input signal, and the acceleration of the vertical canceller's movable mass was used as the output signal. Frequency analysis was processed using MATLAB. One of the experimental results obtained is shown in Figure 5. The values of $\omega_{n v}, K_{v}$, and $\zeta_{v}$ were identified using these results, and the frequency response of the plant model was simulated using Eq. (1) and the above-identified parameters. One of the simulation results obtained is also shown in Fig. 5. Figure 5 shows that the plant analytical model can represent the real characteristics of the vertical canceller accurately. The active vibration-canceling system uses four sets of vertical cancellers, but other cancellers also give experimental and simulation results identical to results obtained here.

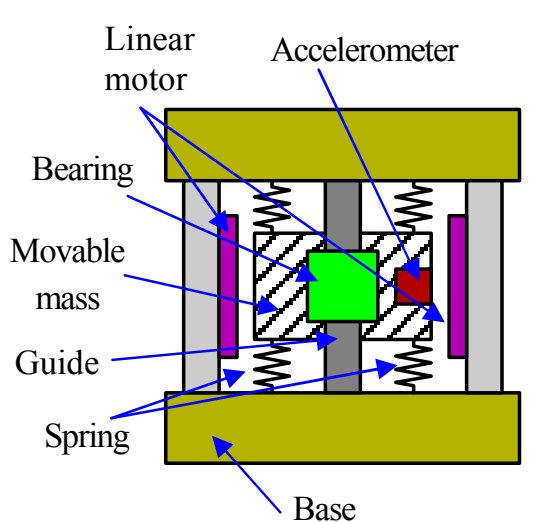

Fig. 4 Vertical canceller
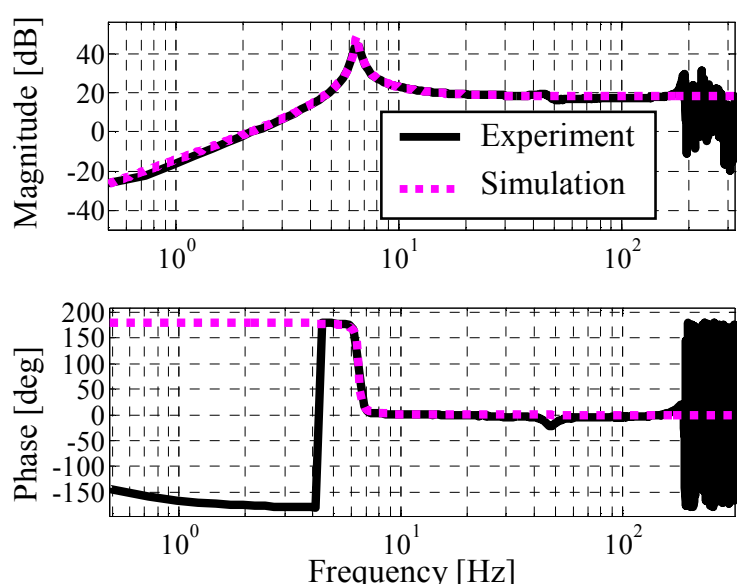

Fig. 5 Frequency response of the vertical canceller 


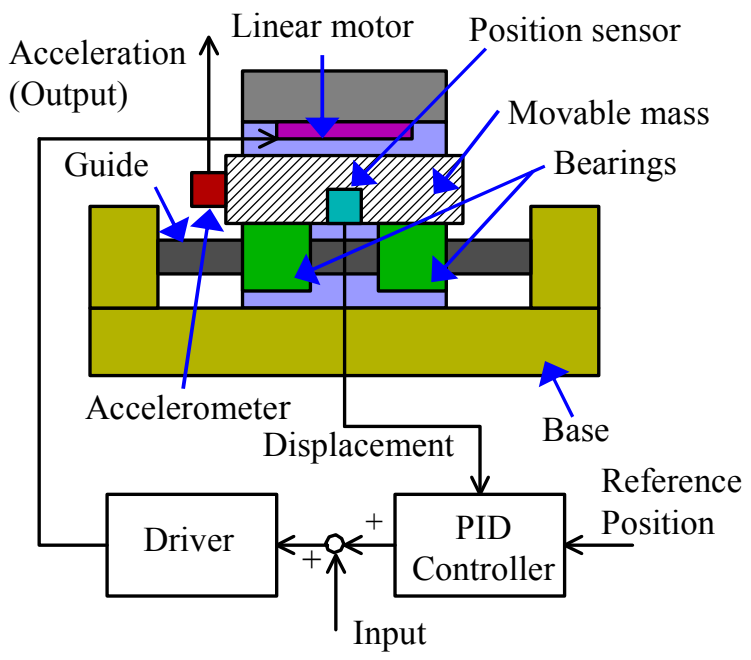

Fig. 6 Horizontal canceller

\subsubsection{Horizontal Canceller}

Figure 6 shows a schematic view of the horizontal canceller. The horizontal canceller generates an inertial force by driving the movable mass, which is guided by a linear guide with a linear motor. The weight of the horizontal canceller's movable mass is about $27 \mathrm{~kg}$. The difference between this and the vertical canceller is that the horizontal canceller does not have a spring supporting the movable mass. The horizontal canceller uses a PID position controller instead of a supporting spring. The developed active vibration-canceling system has just one horizontal canceller. Therefore, the single horizontal canceller must generate a large acceleration to generate the required inertial force. As a result, the horizontal canceller's movable mass requires a long movable span. However, it is difficult to set up a spring so that it properly supports the movable mass over a long movable span. Therefore, the PID position controller is used in place of the spring.

The plant analytical model of the horizontal canceller is derived in order to design the acceleration controller for the horizontal canceller. The plant model of the horizontal canceller consists of the horizontal canceller and the PID position controller, as shown in Fig. 6. The plant analytical model is given as the transfer function, from the input signal for the linear motor driver to the acceleration of the horizontal canceller's movable mass. The frequency response of the horizontal canceller was initially measured. The following values were used as position-controller gains in this experiment. Proportional gain $K_{p}$ was 4 , integral gain $K_{I}$ was 1 and pseudo-derivative compensator gain $K_{D}$ was 1 . The cutoff frequency of the pseudo- derivative compensator was $20 \mathrm{~Hz}$. In the experiment, a sine wave sweeping from 0.5 to $300 \mathrm{~Hz}$ in $120 \mathrm{~s}$ was used as the input signal. This sine-wave signal and control input of the PID controller was input to the linear motor driver. The output signal was the acceleration of the horizontal canceller's movable mass. The frequency response was obtained by processing these signals in the same way as for the vertical canceller. Figure 7 shows the frequency response of the horizontal canceller. Figure 7 also shows that the horizontal canceller exhibits resonance characteristics at about $150 \mathrm{~Hz}$. It is considered that this resonance characteristic is caused by low-stiffness mechanical components such as sheet-metal parts. Even though, the horizontal canceller's plant analytical model can be derived via the PID position controller and the equation of motion for the horizontal canceller. This analytical model did not adequately represent the above-mentioned resonance characteristics. Therefore, a 2nd-order system with natural angular frequency $\omega_{n a}$ and damping ratio $\zeta_{a}$ was added to the horizontal canceller's plant analytical model to represent these resonance characteristics. Finally, the horizontal canceller's plant analytical model $P_{H}$ is given in Equation(2), where $m_{h}$ is the horizontal 

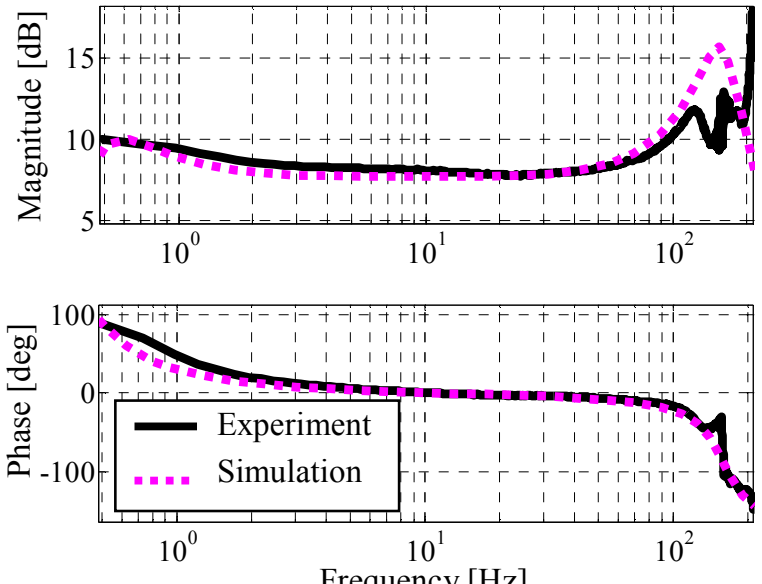

Frequency $[\mathrm{Hz}]$

Fig. 7 Frequency response of the horizontal canceller

canceller's movable mass, $a_{h}$ is the gain of the linear motor, $c_{h}$ is the viscosity coefficient of the bearing, and $T_{D}$ is the time constant of the pseudo-derivative compensator.

$$
P_{H}=\frac{a_{h} s^{3}\left(1+T_{D} s\right)}{\alpha_{4} s^{4}+\alpha_{3} s^{3}+\alpha_{2} s^{2}+\alpha_{1} s+\alpha_{0}} \cdot \frac{\omega_{n a}{ }^{2}}{s^{2}+2 \omega_{n a} \zeta_{a} s+\omega_{n a}{ }^{2}}
$$

where,

$$
\begin{aligned}
& \alpha_{4}=m_{h} T_{D} \\
& \alpha_{3}=m_{h}+c_{h} T_{D} \\
& \alpha_{2}=a_{h} K_{P} T_{D}+a_{h} K_{D}+c_{h} \\
& \alpha_{1}=a_{h} K_{P}+a_{h} K_{I} T_{D} \\
& \alpha_{0}=a_{h} K_{I}
\end{aligned}
$$

The parameters $a_{h}$ and $c_{h}$ were determined via a parameter identification experiment, and $m_{h}$ was measured. The simulation results of the frequency response using the horizontal canceller's plant analytical model are also shown in Fig. 7. These results show that the horizontal canceller's plant analytical model can almost exactly represent the real characteristics of the horizontal canceller.

\section{Design of the Acceleration Control System for the Cancellers}

\subsection{Dual Model Matching Method}

Acceleration controllers for the cancellers were designed using the dual model matching method (DMM). Figure 8 shows the generic control system using DMM. In Fig. $8, r$ is the reference, $d$ is the disturbance, $v$ is the noise, and $y$ is the controlled variable. DMM is a control system design method that can realize the desired two closed-loop characteristics ${ }^{(5)}$. DMM is based on the 2-DOF control system. Therefore, two controllers $C_{r u}$ and $C_{y u}$ governing the characteristics from $r$ to $u$ and the characteristics from $y$ to $u$, respectively, are derived. In order to design the controller using DMM, the transfer function $W_{r y}$ that governs the closed-loop characteristics from $r$ to $y$, and the transfer

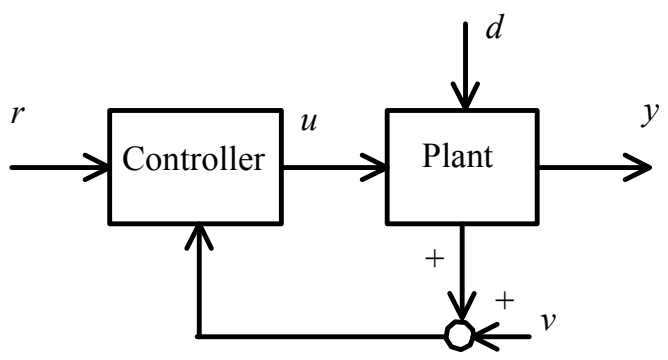

Fig. 8 Dual model matching control system 
function $W_{v y}$ that governs the closed-loop characteristics from $v$ to $y$, or the transfer function $W_{d y}$ that governs the closed-loop characteristics from $d$ to $y$, are required. $W_{r y}, W_{v y}$, and $W_{d y}$ govern the tracking performance, noise rejection characteristics, and disturbance suppression characteristics, respectively. However, these transfer functions are required in order to satisfy the several conditions that can realize a proper controller. In addition, $W_{v y}$ and $W_{d y}$ cannot be independently designed ${ }^{(4)}$. By setting $W_{r y}$ and $W_{v y}$ to satisfy the above conditions, controllers were derived via Equation(3) and Equation(4), where $P_{u y}$ is the transfer function of the plant.

$$
\begin{aligned}
& C_{r u}=P_{u y}{ }^{-1} W_{r y}\left(1+W_{v y}\right)^{-1} \\
& C_{y u}=P_{u y}{ }^{-1} W_{v y}\left(1+W_{v y}\right)^{-1}
\end{aligned}
$$

The reference acceleration tracking performance is very important for the active vibration-canceling system. Therefore, DMM is good controller design method for this system, because DMM can directly design the reference tracking characteristics.

\subsection{Design of the Acceleration Controller for the Vertical Canceller}

The acceleration controller for the vertical canceller was designed via DMM. The design requirements are as follows.

- Realize the required acceleration tracking performance.

- Remove the DC component and noise included in the acceleration sensor signal.

- Prevent the uniform accelerated motion of the movable mass.

To satisfy the above requirements, characteristics from $r$ to $y$ were designated band-pass characteristics, and characteristics from $v$ to $y$ were also designated band-pass characteristics. $W_{r y}$ and $W_{v y}$, used to design the vertical canceller's acceleration controller, were determined considering the above conditions and characteristics as follows.

$$
\begin{aligned}
& W_{r y}=\frac{K_{a} K_{v} s^{3}\left(s-\lambda_{1}\right)\left(s-\lambda_{2}\right)\left(s-\lambda_{3}\right)\left(s-\lambda_{4}\right)\left(s-\lambda_{5}\right)}{\left(s-P_{1}\right)\left(s-P_{2}\right)\left(s-P_{3}\right) \cdots\left(s-P_{7}\right)\left(s-P_{8}\right)\left(s-P_{9}\right)} \\
& W_{v y}=\frac{K_{v} s^{3}\left(\beta_{2} s^{2}+\beta_{1} s+\beta_{0}\right)\left(s-\lambda_{6}\right)\left(s-\lambda_{7}\right)}{\left(s-P_{1}\right)\left(s-P_{2}\right)\left(s-P_{3}\right) \cdots\left(s-P_{7}\right)\left(s-P_{8}\right)\left(s-P_{9}\right)}
\end{aligned}
$$

Here, $K_{a}$ is a constant, $\lambda_{1}-\lambda_{7}$ are zeros, and $P_{1}-P_{9}$ are the poles of the closed-loop transfer function. In the controller design using DMM, by determining these parameters to satisfy the requirements, the controllers are derived from Eq. (3) and Eq. (4). In addition, $\beta_{0}-\beta_{2}$ are also determined through this process.

The performance of the designed acceleration control system was evaluated. In this
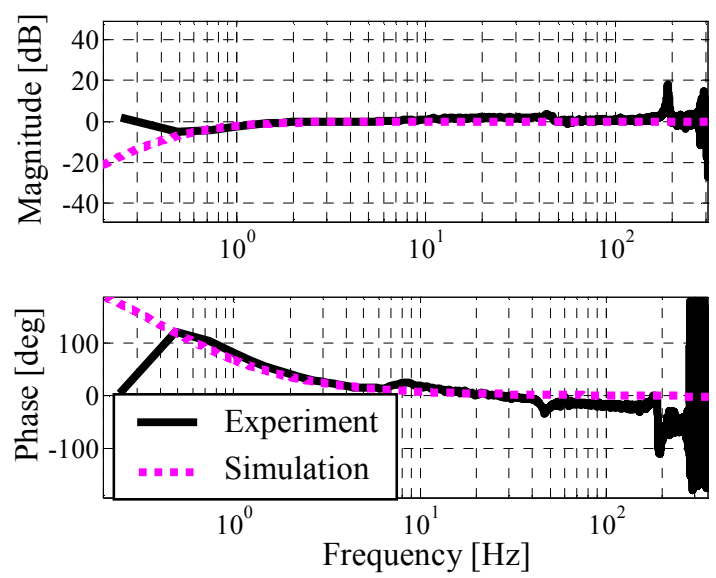

Fig. 9 Acceleration control performance of the vertical canceller 
performance evaluation, the frequency response of $W_{r y}$ (reference tracking performance) was measured and compared with the simulation results. In the frequency response experiment, the sine signal sweeping from 0.5 to $300 \mathrm{~Hz}$ in $120 \mathrm{~s}$ was used as the reference acceleration signal, and the acceleration of the vertical canceller was used as the output signal. Frequency analysis was performed in the same way as in the case of the plant model. The experimental results and simulation results are shown in Figure 9. Figure 9 shows that the designed acceleration controllers have a good acceleration tracking performance. The simulation results also match the experimental results. On the other hand, Fig. 9 also shows that this system has a resonance characteristic at near $200 \mathrm{~Hz}$. However, this resonance characteristic does not give the negative effect to the vibration canceling system, because $200 \mathrm{~Hz}$ is much higher than normally used band width. The active vibration-canceling system uses four sets of vertical cancellers. However, the same results were obtained for the other three sets of the vertical cancellers, not shown in Fig. 9.

\subsection{Design of the Acceleration Controller for the Horizontal Canceller}

The acceleration controller for the horizontal canceller is also designed by DMM. The design requirements are the same as those for the vertical canceller. $W_{r y}$ and $W_{v y}$, which were used to design the horizontal canceller's acceleration controller, are shown as follows.

$$
\begin{aligned}
& W_{v y}=\frac{N_{H} \cdot s\left(s-\beta_{1}\right)\left(s-\beta_{2}\right)\left(b_{5} s^{5}+b_{4} s^{4}+b_{3} s^{3}+b_{2} s^{2}+b_{1} s+b_{0}\right)}{\left(s-P_{1}\right)\left(s-P_{2}\right)\left(s-P_{3}\right) \cdots \cdots\left(s-P_{15}\right)\left(s-P_{16}\right)} \\
& W_{r y}=\frac{N_{H} \cdot N_{r y}^{\prime}}{\left(s-P_{1}\right)\left(s-P_{2}\right)\left(s-P_{3}\right) \cdots \cdots\left(s-P_{15}\right)\left(s-P_{16}\right)}
\end{aligned}
$$

Here, $P_{1}-P_{16}$ are poles of the closed-loop transfer function. $N_{H}$ is a numerator polynomial of the horizontal canceller's plant analytical model. $\beta_{1}$ and $\beta_{2}$ are parameters that can be determined by the designer. $N_{r y}$ ' is a polynomial in $s$ with a degree less than 10th-order to realize a proper controller. By determining these parameters to satisfy the design requirements, controllers are derived from Eq. (3) and Eq. (4) in the design of the controller using DMM. In addition, $b_{0}-b_{5}$ are also determined via this process. The performance of the designed acceleration control system was evaluated. In this performance evaluation, the frequency response of $W_{r y}$ (reference tracking performance) was measured and compared with the simulation results. Frequency analysis was also processed in the same way as in the vertical canceller's case. The results are shown in Figure 10. Figure 10 shows that good acceleration control performance is realized by the designed acceleration controllers. The simulation results also match the experimental results. From these results, we can confirm that the acceleration of the horizontal canceller's movable mass can be
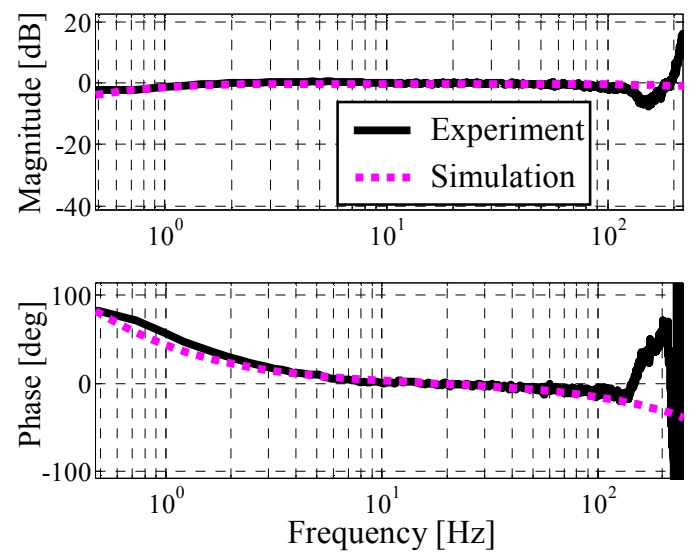

Fig. 10 Acceleration control performance of the horizontal canceller 
controlled as required by the designed acceleration controller.

\section{Evaluation Experiments}

Confirmation experiments for the vibration-canceling effect were performed using experimental apparatus shown in Fig. 11. In these experiments, the performance of the active vibration-canceling system was evaluated while limited to 3-DOF of motion on the plane. Experimental methods and results are shown as follows.

\subsection{Experimental Method}

This subsection describes the vibration-canceling effect via the active vibration-canceling system. Many stage devices are usually driven by trapezoidal velocity signals. However, the inertial force is generated when the stage device is accelerated or decelerated. Therefore, in these experiments, the stage device is driven by triangular reference signals. A triangular reference signal with amplitude of $2 \mathrm{~V}$ and frequency of 10 $\mathrm{Hz}$ was input to the servo driver for the stage device every $2 \mathrm{~s}$. Then, the acceleration on the table and excitation force transmitted to the floor were measured in $60 \mathrm{~s}$ intervals. The vibration-canceling effect was evaluated using the magnitude of these acceleration and excitation force. The acceleration on the table was measured via the accelerometer No.5 (for the horizontal direction), and the accelerometer No.6 and No.7 (for the vertical direction), as shown in Figure 11. The excitation force transmitted to the floor should be directly measured by the force sensor. However, it is difficult to measure the excitation force exactly, because the excitation force is very small compared to the weight of the experimental apparatus. On the other hand, the active vibration isolation device uses air actuators between the table and the floor it is installed on. In addition, the pressure-acceptable area of this air-actuator is constant, even when the air actuator is operational. Therefore, we can assume that the excitation force is proportional to the fluctuation of the air actuator's inner pressure. From the above, the excitation force transmitted to the floor can be evaluated by measuring the fluctuation of the air actuator's inner pressure. Air actuators are installed at each corner of the table. In these experiments, the inner pressure fluctuations of the air actuators No.1 and No.2 (front side of this figure) shown in Fig. 11 were measured. Based on the above method, the effectiveness of the active vibration-canceling system was confirmed by comparing the case of using the active vibration-canceling system with the case of not using the active vibration-canceling system. If the active vibration-canceling system works well, there are no observed effects of the stage device operation on the table or the floor.

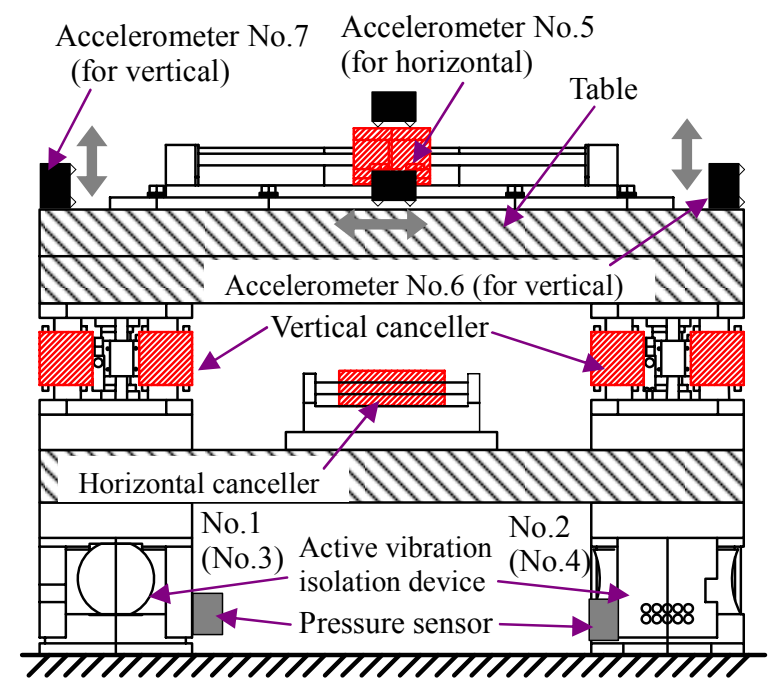

Fig. 11 Sensors arrangement 


\subsection{Experimental Results}

Experimental results are presented below. Figure 12 is a time response of the horizontal acceleration on the table, measured by the accelerometer No.5. Figures 13 and 14 are the time responses of the vertical accelerations on the table, measured by the accelerometers No.6 and No.7. The triangular signal to the stage device was input at about $43 \mathrm{~s}$. These results show that the active vibration-canceling system can sufficiently cancel the effect of the stage device operation. By measuring with the three sets of accelerometers, we can confirm the effectiveness of the vibration-canceling in the horizontal direction of the table,

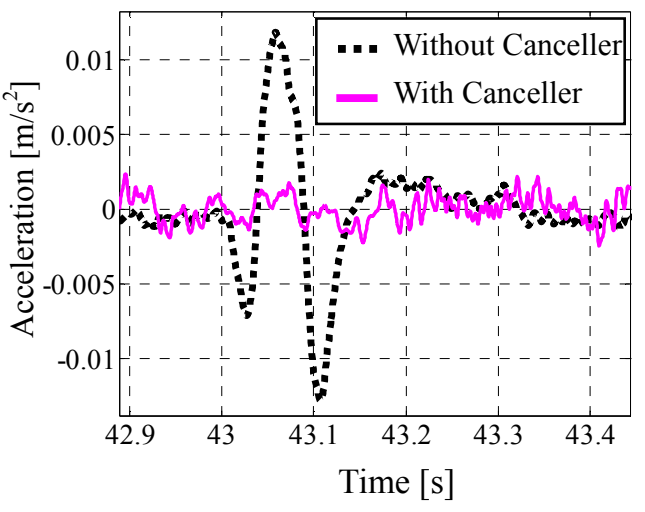

Fig. 12 Time response of the horizontal acceleration on the table (No.5)

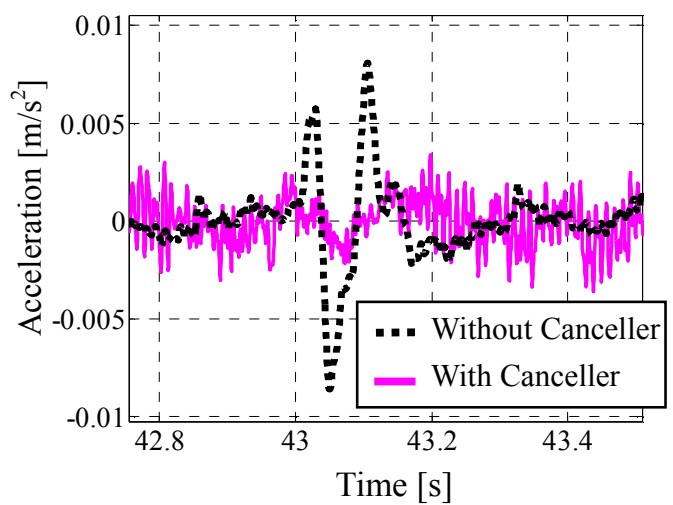

Fig. 13 Time response of the vertical acceleration on the table (No.6)

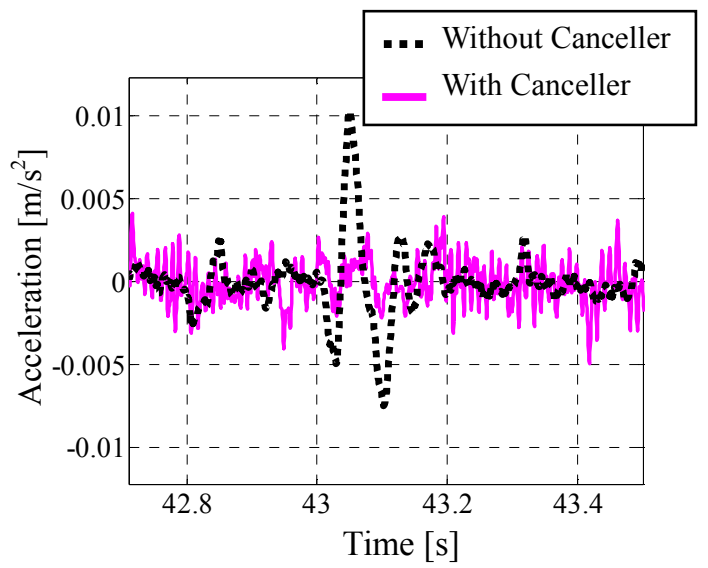

Fig. 14 Time response of the vertical acceleration on the table (No.7)

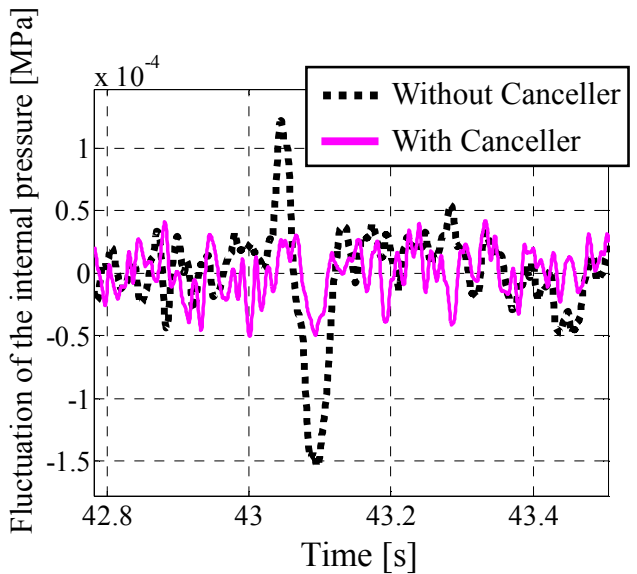

Fig. 15 Time response of the fluctuation of the internal pressure (No.1 device)

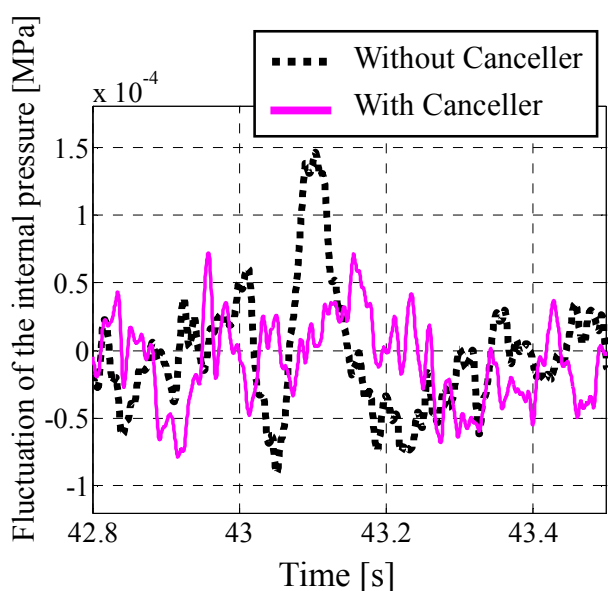

Fig. 16 Time response of the fluctuation of the internal pressure (No.2 device) 
the vertical direction of the table, and the rotary direction of the table. In addition, a high-frequency vibration appears when the active vibration-canceling system is operational. This problem may arise because the high-frequency vibration generated by the stage device overlaps with the reference signal of the canceller. Figures 15 and 16 show the time responses of the air actuator's inner pressure fluctuation. These results were obtained using a $0.5-50 \mathrm{~Hz}$ bandpass filter to remove the sensor noise and DC component from the pressure sensor's signal. These results show that when the active vibration-canceling system is used, the peak value of the excitation force transmitted to the floor can be suppressed to about $30 \%$ of the value without the active vibration-canceling system.

\section{Conclusions}

This paper describes the development of our active vibration-canceling system. The damping force of the active vibration isolation device causes the floor it is installed upon to vibrate. As a result, other precision devices installed on the same floor are negatively affected. Our active vibration-canceling system, which uses an inertial-force generator, "canceller" can solve this problem. In addition, we have confirmed via several experiments that the stage device's reaction force, which is transmitted to the table, is cancelled successfully.

\section{References}

(1) Yasuda M., and Ikeda M., Double-active Control of Microvibration Isolation Systems to Improve Performance, Transactions of the Japan Society of Mechanical Engineers, series $C$, Vol. 59-No.562 (1993), pp.1694-1701.

(2) Fujita T., Tagawa Y., Kajiwara K., Yoshioka H,. Takeshita A., and Yasuda M., Active Microvibration Control system Using Piezoelectric Actuator, Transactions of the Japan Society of Mechanical Engineers, series C, Vol. 59-No.559 (1993), pp.753-739.

(3) Yasuda M., Osaka T., and Ikeda M., Study of Active Isolation System with Feedforward Control, Transactions of the Japan Society of Mechanical Engineers Series C, Vol.58, No.552(1992), pp.2381-2387.

(4) Tagami M., Yamashita T., Tagawa Y., Yasuda M., Hora H., Endo K., and Noguti Y., Development of an Active Vibration Canceling System Using a Stage Driving Signal, Proceedings of the MoVic2006, CD-ROM, Korea (2006).

(5) Tagawa Y., Seino Y., and Yasuda M., Study of 6-DOF Microvibration Control Using Feedfoward Control, Transactions of the Japan Society of Mechanical Engineers Series C, Vol. 63-No.608 (1997), pp.1081-1087. 